\title{
A systematic review of economic evaluations of CHW interventions aimed at improving child health outcomes
}

\author{
L. Nkonki ${ }^{*}$, A. Tugendhaft ${ }^{2}$ and K. Hofman ${ }^{2}$
}

\begin{abstract}
Evidence of the cost-effectiveness of community health worker interventions is pertinent for decision-makers and programme planners who are turning to community services in order to strengthen health systems in the context of the momentum generated by strategies to support universal health care, the post-2015 Sustainable Development Goal agenda.

We conducted a systematic review of published economic evaluation studies of community health worker interventions aimed at improving child health outcomes. Four public health and economic evaluation databases were searched for studies that met the inclusion criteria: National Health Service Economic Evaluation Database (NHS EED), Cochrane, Paediatric Economic Evaluation Database (PEED), and PubMed. The search strategy was tailored to each database. The 19 studies that met the inclusion criteria were conducted in either high income countries (HIC), low- income countries (LIC) and/or middle-income countries (MIC). The economic evaluations covered a wide range of interventions. Studies were grouped together by intended outcome or objective of each study. The data varied in quality. We found evidence of cost-effectiveness of community health worker (CHW) interventions in reducing malaria and asthma, decreasing mortality of neonates and children, improving maternal health, increasing exclusive breastfeeding and improving malnutrition, and positively impacting physical health and psychomotor development amongst children.

Studies measured varied outcomes, due to the heterogeneous nature of studies included; a meta-analysis was not conducted. Outcomes included disease- or condition -specific outcomes, morbidity, mortality, and generic measures (e.g. disability-adjusted life years (DALYs)). Nonetheless, all 19 interventions were found to be either cost-effective or highly cost-effective at a threshold specific to their respective countries.

There is a growing body of economic evaluation literature on cost-effectiveness of CHW interventions. However, this is largely for small scale and vertical programmes. There is a need for economic evaluations of larger and integrated CHW programmes in order to achieve the post-2015 Sustainable Development Goal agenda so that appropriate resources can be allocated to this subset of human resources for health. This is the first systematic review to assess the cost-effectiveness of community health workers in delivering child health interventions.
\end{abstract}

Keywords: Community health worker, Economic evaluations, Child health, Cost-effectiveness

\footnotetext{
* Correspondence: Inkonki@sun.ac.za

${ }^{1}$ Centre for Health Systems and Services Revision, Division of Community Health, Faculty of Medicine and Health Sciences, Francie van Zijl Rylaan/ Drive, Tygerberg 7505, South Africa

Full list of author information is available at the end of the article
} 


\section{Background}

Community services are seen as key for strengthening health systems in the context of the momentum generated by strategies for universal access to health care and the post-2015 Sustainable Development Goal agenda. Thus, the question of effectiveness and cost-effectiveness of community health worker $(\mathrm{CHW})$ interventions is pertinent for decision-makers and programme planners. The choice of which CHW service or services to deliver needs to be underpinned by evidence of both effectiveness and cost-effectiveness. A substantial body of evidence on CHW intervention effectiveness exists using varied methodologies, time frames, and scope [1-7], but all six [1-6] reviews consistently highlight the scarcity of economic evaluations.

Evidence on costs and cost-effectiveness of $\mathrm{CHW}$ intervention is crucial for decision-making. CHW interventions are expected to have favourable economic evaluations because they are perceived to be cheap, fast, and easy. Largely because training of CHWs is significantly shorter than training of other health professionals (e.g. medical doctors and nurses), they extend coverage to geographically hard-to-reach populations and they are often paid a stipend or work as volunteers. In spite of all these factors, CHW programmes in many countries in the 1970s and 1980s were abandoned as they failed to realize the potential demonstrated in several initiatives led by nongovernmental organizations and in national programmes such as China's "barefoot doctors".

Several reviews [1-7] have reported that CHWs undertake a wide variety of tasks in primary and public health $[4,5]$ and CHWs deliver interventions in primary health care including nutrition, maternal and child health, malaria control, tuberculosis (TB) control, HIV/AIDS prevention and control, mental health, and non-communicable disease. A Cochrane review of $\mathrm{CHW}$ interventions identified 107 randomized control trials (RCTs) which showed promising benefits, compared to usual care, in increasing immunization uptake in children, improving breastfeeding rates until 6 months, reducing neonatal mortality, and improving pulmonary TB care rates. The review also reported that CHWs reduce child morbidity and child mortality, maternal mortality, and increase the likelihood of seeking care for childhood illness [8].

In 2009, a systematic review of economic evaluations of CHWs delivering vaccination programmes found only three studies that matched the inclusion criteria [9]. More recently, a non-systematic review on effectiveness identified nine specific areas in which CHWs are costeffective: specific nutrition intervention, communitybased therapeutic care for children with severe acute malnutrition, pneumonia control, diarrhoea prevention or treatment, malaria control, perinatal/neonatal care programmes, HIV/AIDS control in children, child survival programmes, and comprehensive primary health care with a community-based component [10]. Because this was not a systematic review, there is potential bias introduced during the search, selection of the studies, and interpretation of studies stages.

Systematic reviews aim to reduce bias in the estimation of the effects of a policy option by identifying all relevant studies, selecting those that meet explicit criteria, appraising their quality, and synthesizing the results using a transparent process. To address the current gap in knowledge regarding cost-effectiveness of $\mathrm{CHW}$ services for reducing child and maternal mortality, we have conducted a systematic review of economic evaluations of published economic evaluation studies aimed at improving child health outcomes.

\section{Methodology}

\section{Search methods for identification of studies}

We searched the following public health and economic evaluation databases for studies conducted globally on community health worker (CHW) interventions to improve child health outcomes: National Health Service Economic Evaluation Database (NHS EED), Cochrane, Paediatric Economic Evaluation Database (PEED), and PubMed. The search strategy was tailored to each database.

The functional definition of CHW was used, that being a member of the community who has received some training to promote health care or who carries out some health care services, but is not a professional. That definition covers a diverse cadre of workers, and at least 10 terms were used during searches as indicated in Additional file 1.

A combination of search terms was used in each of the databases, and these are shown in Additional file 1 . We also searched for specific interventions such as breastfeeding, sanitation, hand-washing, immunization, and kangaroo mother care. We searched for studies conducted between 1980 and 2014.

\section{Study eligibility}

The key criterion for inclusion was that the study intervention included CHWs and an economic evaluation of the intervention was performed, with no publication restriction date. The review included full economic evaluations as they provide information on the relative trade-offs between the effect of the intervention on costs and outcomes and hence the most relevant information for health care decision-making [11]. Economic evaluations include cost-minimisation analysis (CMA $)^{1}$, costeffectiveness analysis $(\mathrm{CEA})^{2}$, cost-utility analysis $(\mathrm{CUA})^{3}$, cost-benefit analysis $(\mathrm{CBA})^{4}$, and cost consequence $(\mathrm{CC})^{5}$. All economic evaluations have two components; the first component is a costing analysis ${ }^{6}$ and the second component measures effects. All the above-mentioned economic 
evaluation studies (CMA, CEA, CUA, CBA, and CC) measure costs identically, but they differ in how they measure effects.

We excluded studies that (1) were published in languages other than English; (2) were not original studies; (3) did not provide any costing detail; (4) were designed such that CHWs were just one of several interventions being compared and it was difficult to distinguish the effect and costs of the $\mathrm{CHW}$ intervention.

\section{Study selection}

Two reviewers, with experience in health economics, were involved in the review process. One reviewer independently assessed the potential relevance of all titles and abstracts. Full-text copies of the articles identified as potentially eligible for inclusion were retrieved by the first reviewer. Assessment of the eligibility of interventions can vary between reviewers. Therefore, each full paper was evaluated independently for inclusion by both reviewers. Disagreements on the full-text articles were resolved through discussion between whichever two review authors the article was assigned to and, where necessary, by consulting a third author from the core team for an independent assessment. The final reviewing and writing of the summary and conclusions were done by the second reviewer.

\section{Data extraction}

Two reviewers independently and in duplicate extracted data from each included study. A standard form was developed to extract descriptive and outcome. The form was based on a set of questions developed by Pegurri et al. [12] and Corluka et al. [9]. We have also added other questions based on our experience of $\mathrm{CHW}$ interventions such as retention of CHWs. The form's appropriateness was assessed by piloting the form on selected full economic evaluation articles. The information that was extracted included:

1. Study area and population.

2. Perspective of the economic evaluation in other words was the economic evaluation conducted from a societal, provider, or patient perspective.

3. Timing of the economic evaluation.

4. Type of intervention delivered by CHW.

5. CHW role.

6. Training.

7. CHW retention (number of CHWs who have ceased to work as $\mathrm{CHW}$ in the project).

8. Incentives (financial and non-financial).

9. Duration of the intervention.

10. Study-type economic evaluation (e.g. is it a cost-effectiveness, cost analysis).

11. Comparator(s).
12. Costing approach.

(a) Type of costs collected: financial or economic costs.

13. Outputs (unit costs).

14. Scale-up/operational scenarios (costs of replication/ expansion).

15. Measure of effectiveness, e.g. as described by the authors of the reviews.

16. Economic evaluation outcomes (cost-effectiveness or cost utility analysis).

17. Funder.

It was not feasible to contact study authors to obtain any missing information.

\section{Results}

\section{Description of studies}

A total of 1610 titles and abstracts, written in English, were identified as shown in Fig. 1. Two hundred eightyseven articles (excluding duplicates) were selected for abstract evaluation. An additional 18 records were identified from bibliographic searches. Eighty-three articles were considered potentially eligible for inclusion and full-text articles were obtained; 19 of these met the inclusion criteria (Tables 1 and 2).

\section{Setting}

The majority of economic evaluation studies took place in LMICs. Seven studies were conducted in sub-Saharan Africa: two in Tanzania [13, 14], two in Ghana [15, 16], one in South Africa [17], one in Burkina Faso [18], and one in Uganda [19]. Five studies were undertaken in Asia: three in Pakistan [20-22], one in Nepal [23], and one in Bangladesh [24]. Three studies were done LAC (Latin America and the Caribbean): one in Ecuador [25], one in Chile [26], and one in Jamaica [27]. Four of the 19 studies were performed in HIC countries: three in the United States of America, two of which were targeted to low-income populations [28-30], and one was done in the United Kingdom [31].

\section{Cost-effectiveness of the interventions}

Economic evaluations of $\mathrm{CHW}$ interventions aimed at improving child health outcomes cover a wide range of interventions. Hence, we grouped together studies by intended outcome or objective of each study, as listed below. The three remaining studies were extremely diverse and could not be usefully grouped. Sixteen out of the 19 included studies were targeted at a specific outcome, and the remaining three had several health outcome goals.

Economic evaluations can be conducted alongside a primary epidemiological study, for instance alongside a randomized controlled trial or cohort study. The source 


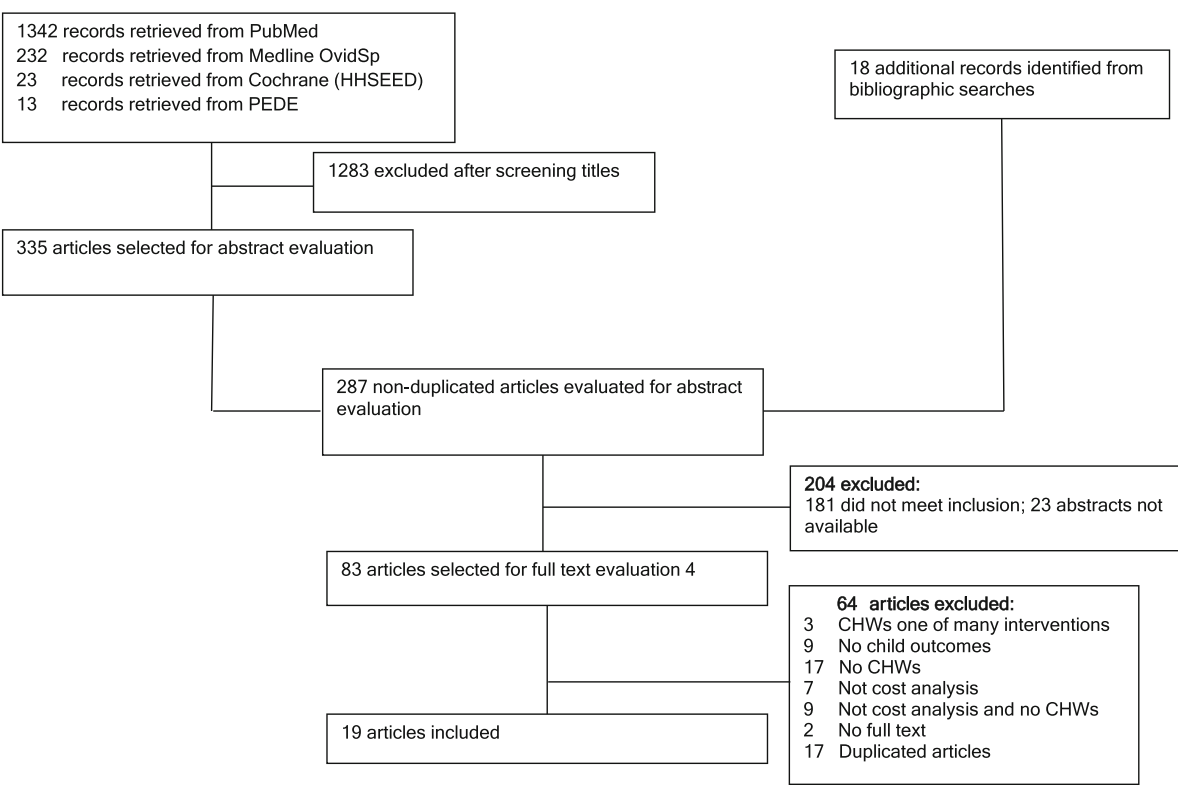

Fig. 1 Flowchart showing the search, selection, and inclusion of studies

of effectiveness data for an economic evaluation is not limited to only one primary study. Effectiveness data can also be collected from synthesizing published effectiveness studies [32]. The economic evaluations reviewed below included primary evaluations and piggyback studies, as well as studies based on previously published literature of costs and effectiveness data (Table 3).

\section{Economic evaluations of $\mathrm{CHW}$ interventions aimed at reducing malaria in children}

Setting Five $[13-16,18]$ studies were conducted in subSaharan Africa. Two studies were conducted from a provider's perspective $[14,18]$ and three from a societal perspective $[13,15,16]$.

Description of interventions The interventions were aimed at both community diagnosis and treatment of malaria. Malaria treatment included treatment for febrile and non-febrile children, many from a single dose of rectal antibiotics to 3 days of medication bimonthly.

The cost-effectiveness of the interventions was measured using generic economic evaluation measures, mortality, disease- or condition-specific measures, and intervention process measures. Three studies $[13,14,16]$ measured cost-effectiveness using disability-adjusted life years (DALYs). DALYs combine years of life lost because of premature death with years of life lived with disability in one outcome measure. One study used years of life lost (YLL) [13]. Two studies measured deaths averted $[14,16]$. Other measures of cost-effectiveness included intervention uptake [14, 18], referral compliance [14], anaemia [13], malaria cases averted [15, 16], adequacy of length of treatment [18], and appropriate treatment [18]. All studies aimed at reducing malaria in children were found to be cost-effective.

Table 1 Economic evaluation studies by region

\begin{tabular}{lll}
\hline Developed countries & Developing countries & $\begin{array}{l}\text { Developing countries } \\
\text { Within sub-Saharan Africa }\end{array}$ \\
\hline Morrell et al. 2006 (United Kingdom) & Gowani et al. 2014 [21] (Pakistan) & Tozan, 2010 [14] (Tanzania) \\
Pugh et al. 2002 [29] (USA) & Barzgar et al. 1997 [20] (Pakistan) & Gonzalez, 2000 [13] (Tanzania) \\
Frick et al. 2012 [28] (USA) & Hafeez et al. 2011 [22] (Pakistan) & Conteh et al. 2010 [15] (Ghana) \\
Margellos-Anast, 2012 [30] (USA) & Borghi, 2005 [23] (Nepal) & Nonvignon et al. 2013 (Ghana) \\
& Puett et al. 2013 [24] (Bangladesh) & Desmond et al. 2008 [17] (South Africa) \\
& San Sebastian et al. 2001 [25] (Ecuador) & Pagnoni et al. 1997 [18] (Burkina Faso) \\
& Aracena et al. 2009 [26] (Chile) & Chola, 2011 [19] (Uganda) \\
\hline
\end{tabular}




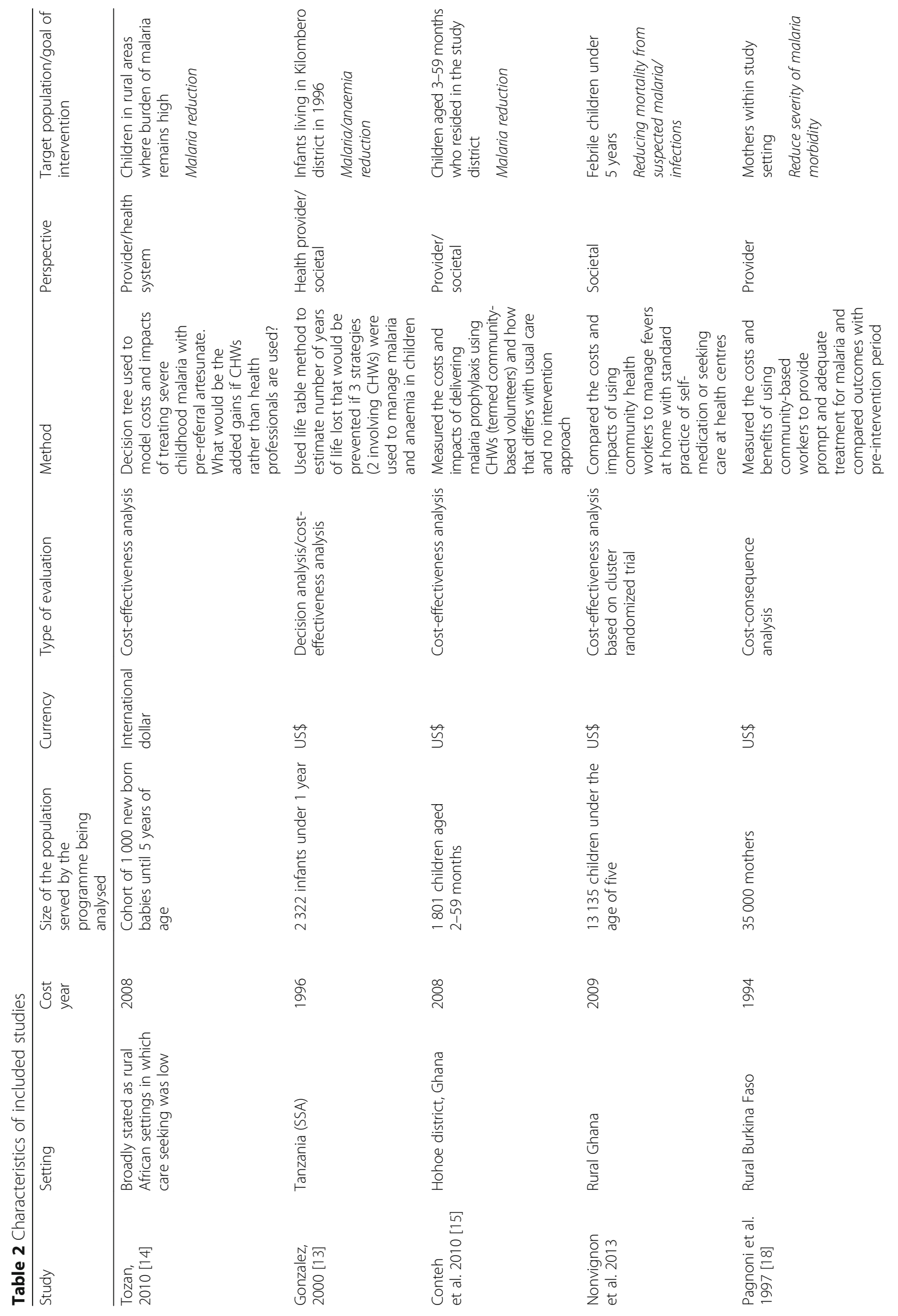




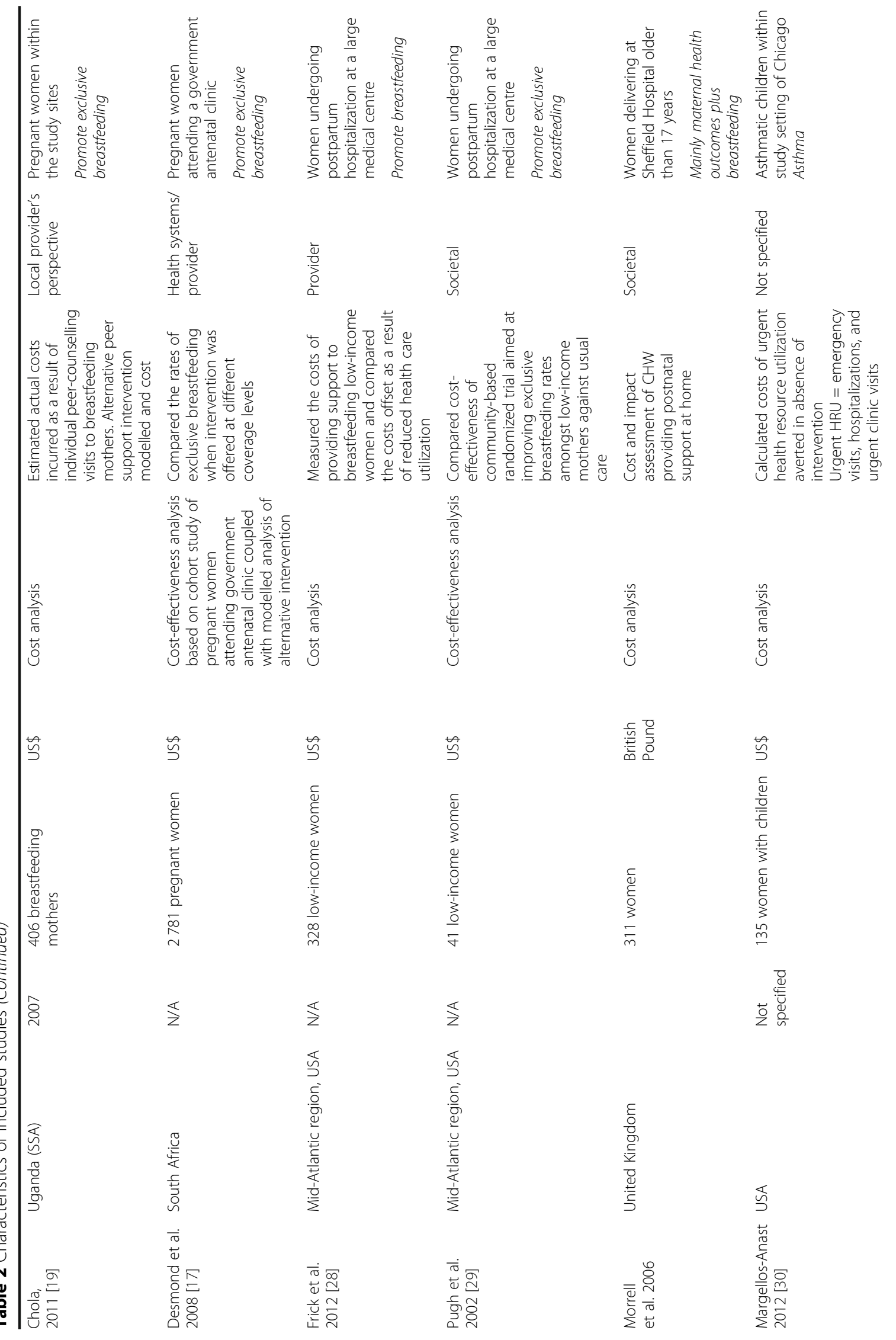




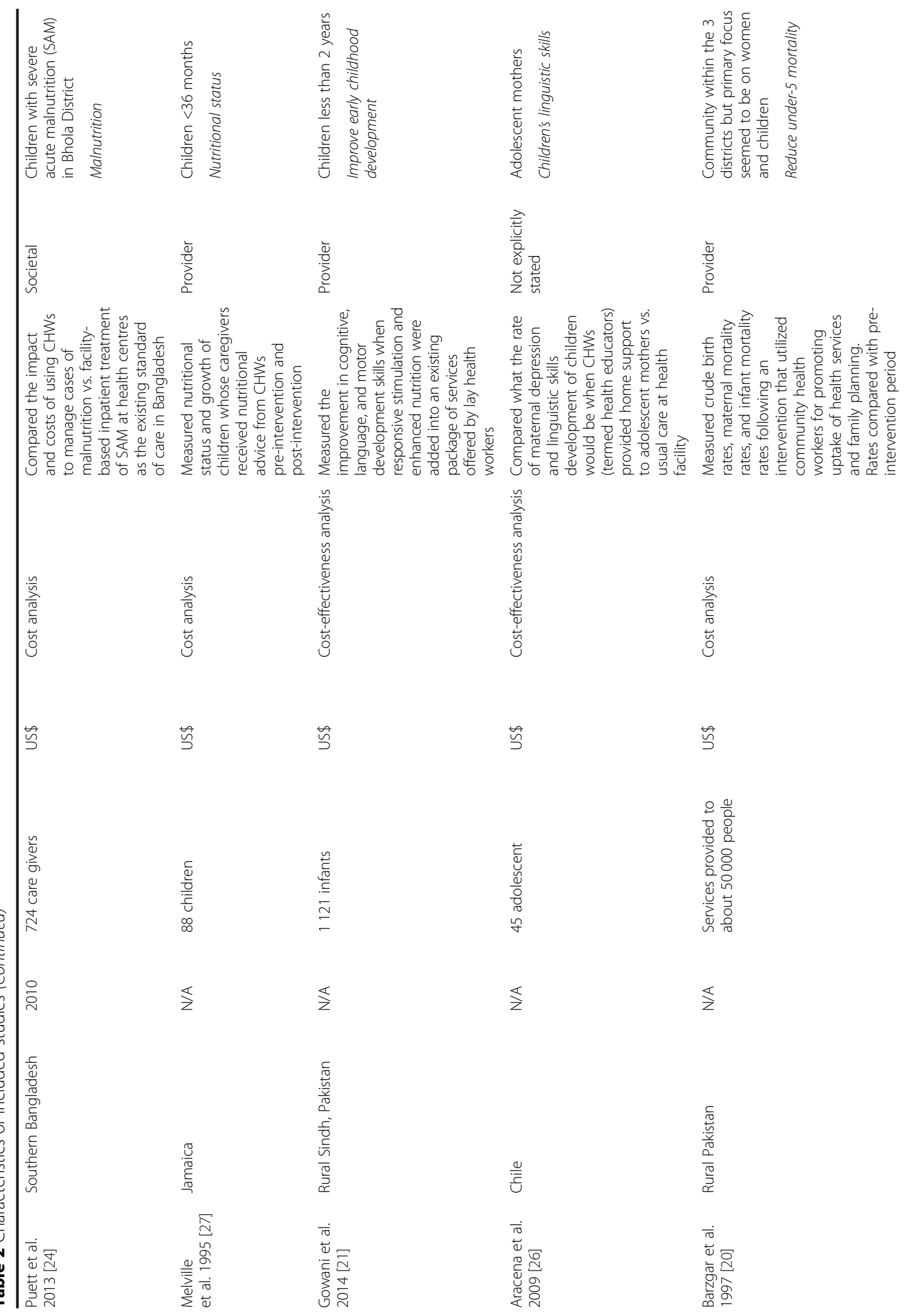




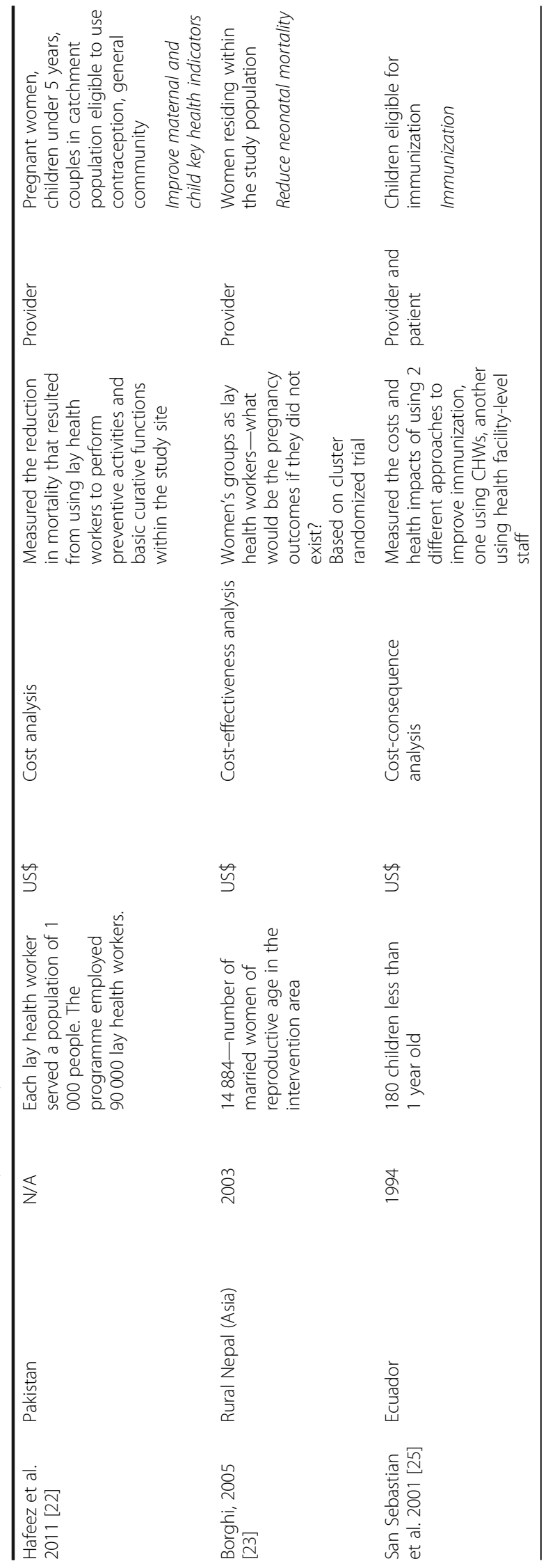




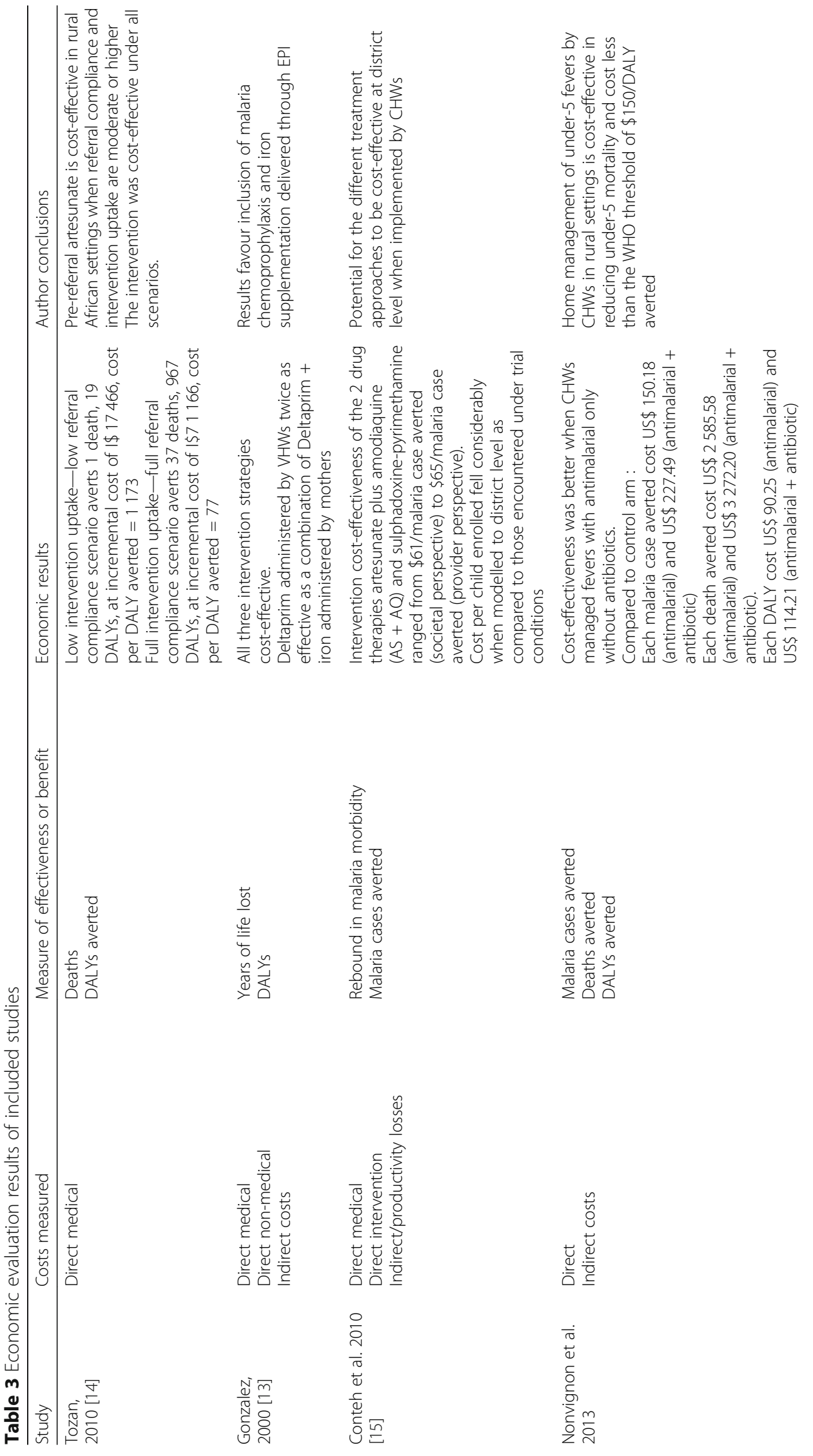




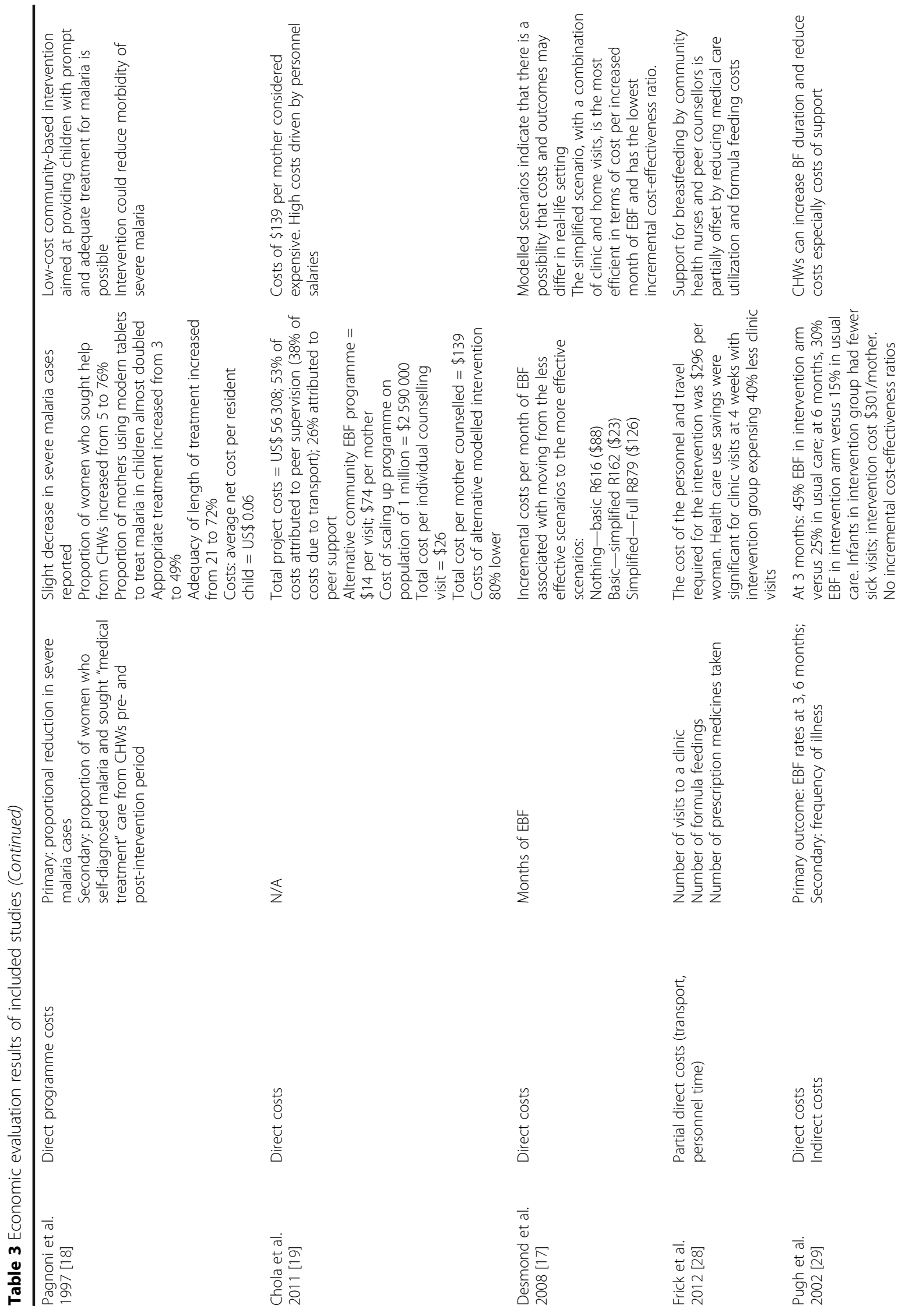




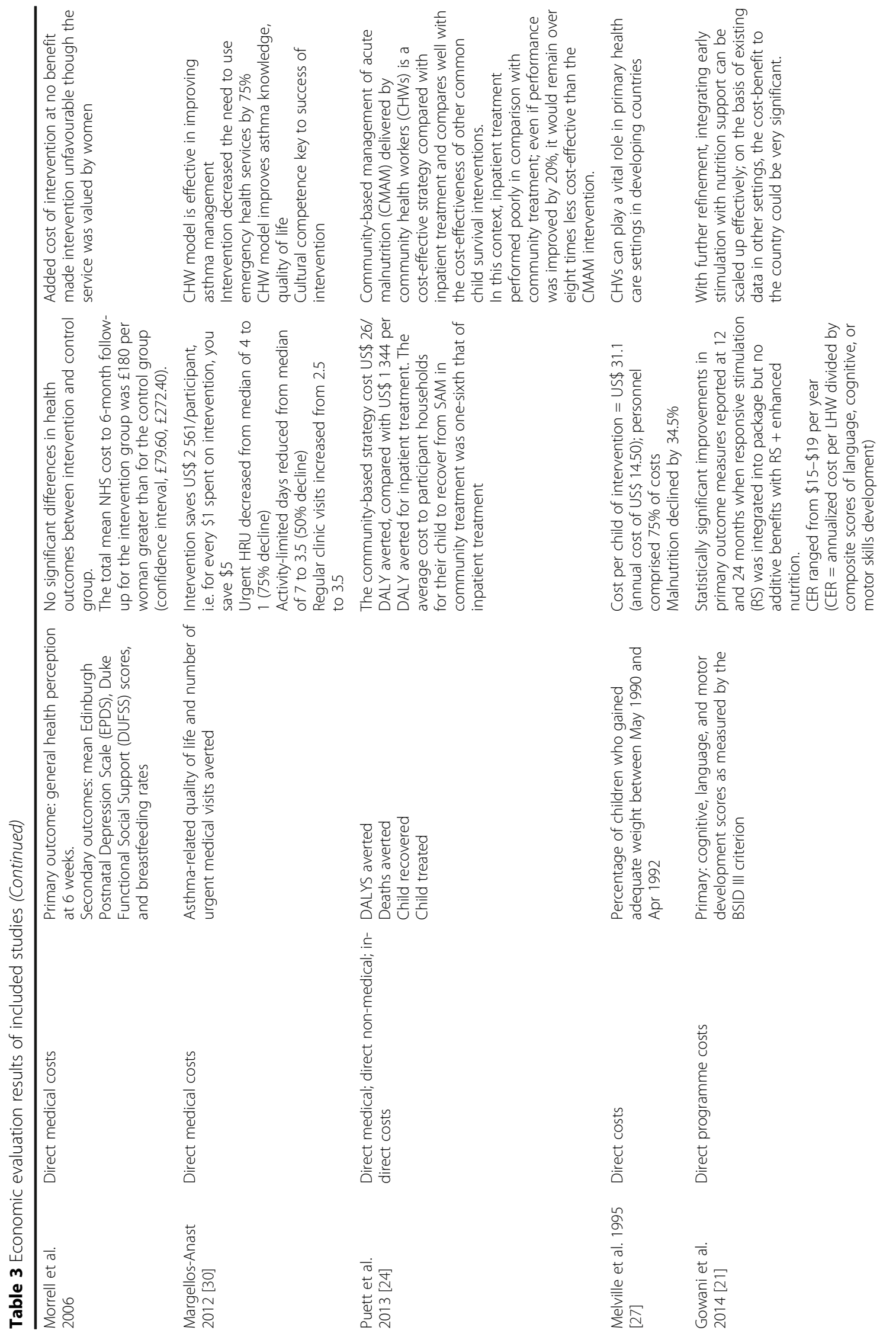




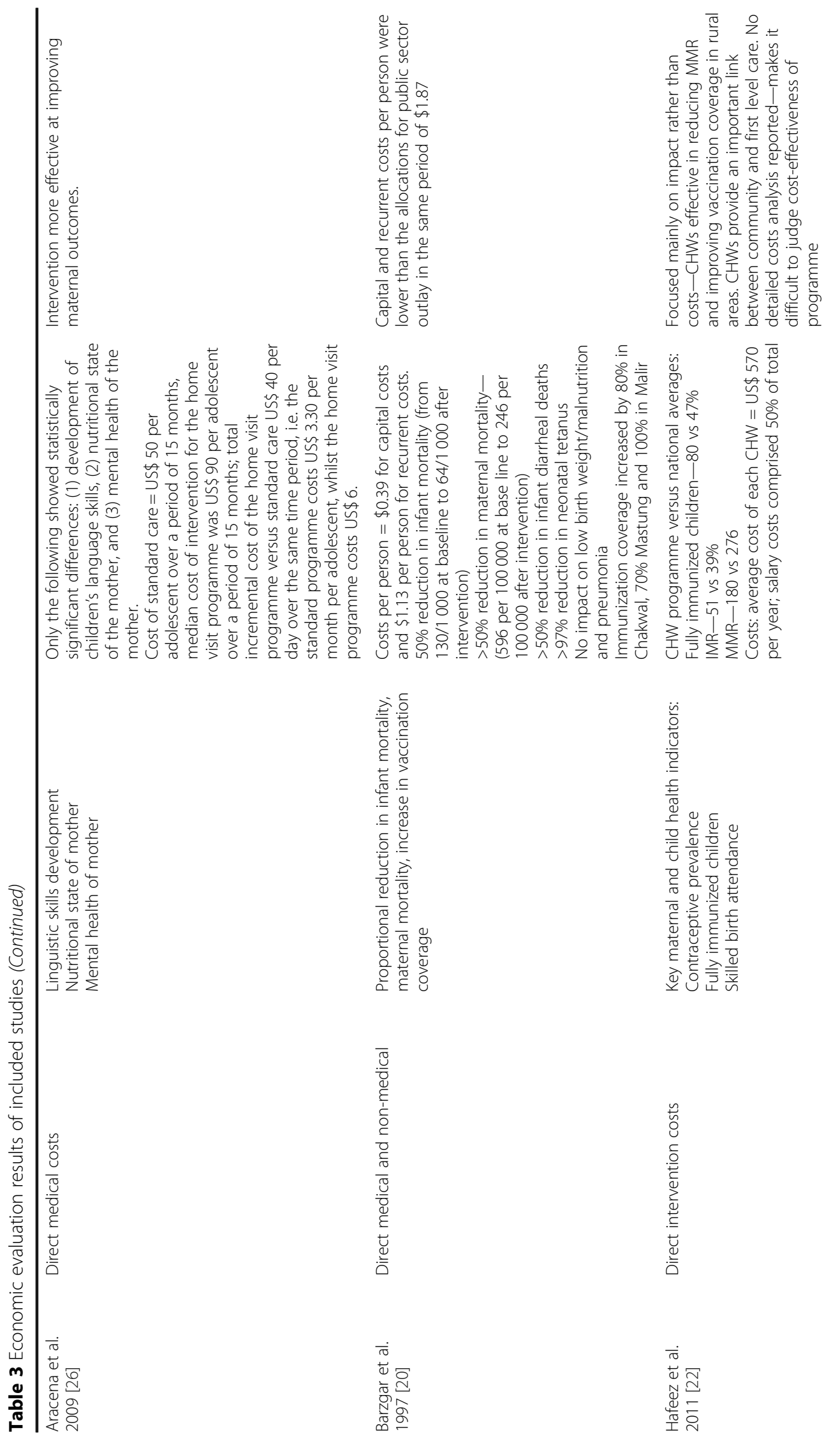




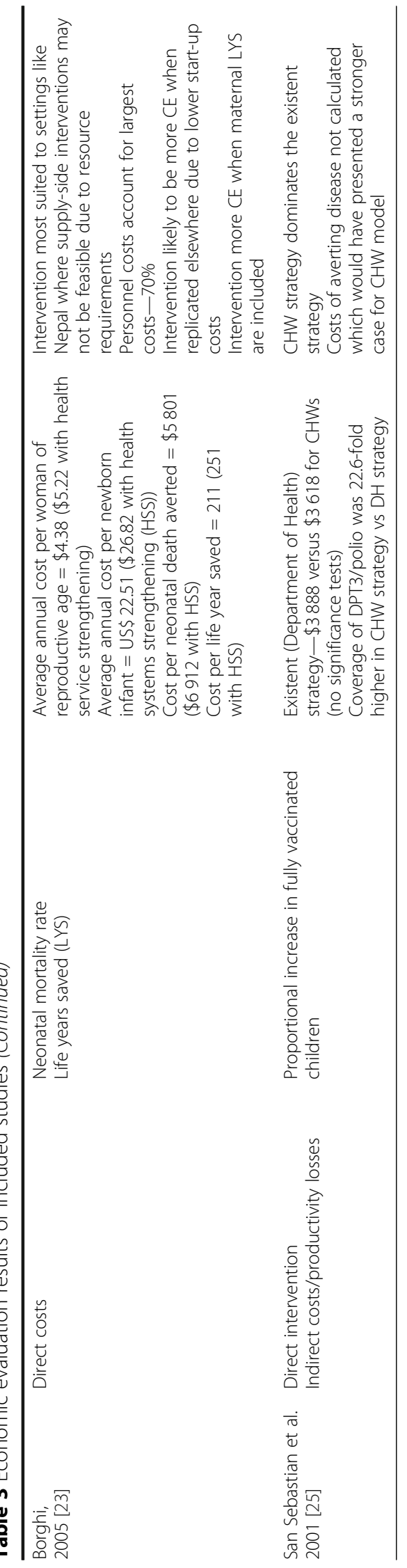


Results The intervention of one dose of rectal artesunate by a CHW to a child with suspected severe malaria alongside referral advice to caregivers was found to be cost-effective [14]. The intervention was estimated to avert 19 DALYs (95\% CI 16-21) at a cost of $\$ 1173$ per DALY averted when the uptake and compliance were both at $25 \%$. When the uptake and compliance were both $100 \%$, the intervention could avert 967 DALYs (95\% CI 884-1050) at a cost of $\$ 77$ per DALY averted.

The three intervention strategies (Deltaprim and iron, Deltaprim, iron) for the prevention of severe anaemia and malaria in infants were found to be cost-effective compared to standard case management [13]. For the prevention of severe anaemia and from the perspective of the health provider, the cost-effectiveness ratios were, respectively, US\$ 8 , US $\$ 9$, and US\$ 21 per DALY for malaria chemoprophylaxis with Deltaprim and iron, Deltaprim alone, and iron alone. For malaria prevention, Deltaprim and iron cost US\$ 9.7 per DALY and Deltaprim alone cost US\$10.2 per DALY. From a sociocultural perspective, the cost-effectiveness ratios ranged from US\$ 9 to US\$ 26 for severe anaemia prevention and US\$ 11 to US\$ 12 for the prevention of clinical malaria.

The two strategies of home management of under-five fevers in Ghana using antimalarial-only artesunateamodiaquine (AAQ) and combined treatment using antimalarial and antibiotics AAQ and artesunateamodiaquine-amoxicillin (AMX) [16]. The cost per anaemia case averted was US\$150.18 for AAQ and US\$ 227.49 for AAQ and AMX, and the cost per death averted was US\$2585.58 for AAQ and US\$3272 for AAQ + AMX. Cost per DALY averted were US\$ 90.25 for AAQ and US\$114.21 for AAQ and AMX.

Intermittent preventive treatment for malaria in children (IPTc) was shown to be cost-effective [15]. During the intervention period, artesunate (AS) and amodiaquine (AQ) monthly was the most cost-effective IPTc drug regimen at US\$ 67.77 (61.71-74.75, 95\% CI) per malaria case averted based on intervention costs only, US\$ 64.93 (58.92-71.92, 95\% CI) per malaria case averted once the provider cost savings are included, and US\$ 61.00 (54.98, $67.99,95 \% \mathrm{CI}$ ) when direct household cost savings are also taken into account. Sulphadoxine-pyrimethamine (SP) bimonthly was US\$105.35 (75.01-157.31, 95\% CI), and AS and AQ bimonthly was US\$ 211.80 (127.05-399.14, 95\% CI) per malaria case averted based on intervention costs only. The incidence of malaria in the postintervention period was higher in children who were less than 1 year old when they received AS and AQ monthly compared to the placebo group leading to higher costeffectiveness ratios when 1-year follow-up is included.

A community-based programme to provide prompt and adequate treatment of presumptive malaria in children was shown to be effective and less costly [18].
The proportion of women who sought help from CHWs increased from 5 to $76 \%$. And the proportion of mothers using modern tablets to treat malaria in children almost doubled. Appropriate treatment increased from 3 to $49 \%$. Adequacy of length of treatment increased from 21 to $72 \%$. The average net cost per resident child was US\$ 0.06 .

\section{Economic evaluations of CHW interventions aimed at promoting exclusive breastfeeding}

Setting Five studies were conducted from a variety of settings: South Africa [17], Uganda [19], United Kingdom [31], and two from the USA [28, 29]. Of the five studies, two were conducted from a societal perspective $[29,31]$.

Description of intervention The main purpose of these interventions was to promote exclusive breastfeeding during both the antenatal period and postpartum. In these studies, mothers received individual support at home. The intensity (i.e. the number of visits per mother) of these visits varied substantially. The number of planned visits in the various studies were 3 [28, 29], 5 [19], 10 [31], and 18 [17]. Two studies included a facility-based intervention. In one study, this was in a form of group education for pregnant mothers during a facility antenatal care visit $[17,28]$. In the economic evaluation conducted by Frick et al. [28], women received telephonic support in addition to the hospital and home visit support.

Results The cost-effectiveness of the interventions was measured using natural units, in this instance breastfeeding rates [33], months of exclusive breastfeeding (EBF) [17], cost per week of EBF [19], and frequency of illness in infants [29]. A few studies measured intermediate outcomes such as cost per mother $[19,28]$, per individual counselling visit [19], and per clinic [28].

An economic evaluation of support to mothers through individual counselling by $\mathrm{CHW}$ increased $\mathrm{EBF}$ prevalence. The cost per mother counselled was US\$ 139 and cost per visit was US\$26. The cost per week of EBF was US\$ 15 at 12 weeks postpartum [19].

Postnatal support for women at home showed no significant difference of breastfeeding rates at 6 weeks [33]. The total mean NHS cost to 6-month follow-up for the intervention group was $£ 180$ per woman greater than for the control group ( $£ 79.60-£ 272.40,95 \% \mathrm{CI}$ ).

An intensive intervention of individual breastfeeding support for mothers was cost-effective and also more costly compared to usual care [17]. The cost per supported month of EBF was US\$41, cost per increased month of EBF was US $\$ 48$, and the incremental costeffectiveness ratio was US\$ 126 .

An intervention comprising of hospital, home visit, and telephone support for 6 months after delivery 
increased duration in low-income women compared to usual care [29]. At 6 months, 30\% of infants were exclusively breastfed (EB) in the intervention arm compared to $15 \%$ in usual care. Infants in the intervention group had fewer sick visits. The cost per mother was US\$ 301 . No incremental cost-effectiveness ratios were calculated.

This intervention conducted in the USA was aimed at increasing breastfeeding rates at 6 weeks and reported reduced medical care utilization and formula feeding costs [28]. The cost per woman was US\$296. Health care use savings were significant for clinic visits at 4 weeks with the intervention group experiencing $40 \%$ less visits.

\section{Economic evaluations of $\mathrm{CHW}$ interventions aimed at reducing asthma amongst children}

Setting In this intervention conducted in the USA, CHWs worked as part of a multidisciplinary team of paediatric pulmonologists, epidemiologists, and intervention coordinators [30]. The study was conducted from a provider perspective.

Description of intervention CHWs did not have prior training on asthma management and were trained for 5 days. As members of the community, they were well acquainted with cultural norms and practice of the target community. The training was conducted by a certified asthma educator and included asthma-related topics, asthma medication and devices, asthma triggers, asthma trigger avoidance and low-cost ways of reducing triggers, and warning signs of an asthma episode and asthma severity. CHWs also received ongoing training.

The CHW conducted four home visits during a 6-month period with participating family and facilitated the establishment of relationship between the family and primary care provider.

Results The efficiency of the asthma control programme was assessed using a cost-savings analysis. Primary outcomes for the intervention included asthma symptoms, asthma-related health resource utilization (HRU) and activity-limited days, and asthma caregiver-related quality of life.

Symptom frequency was reduced by $35 \%$, and urgent health resource utilization was reduced by $75 \%$ between the pre- and post-intervention periods. Parental quality of life also improved by a level that is both clinically and statistically significant. The intervention resulted in cost savings; for every US\$ 1 spent on intervention, US\$ 5.58 is saved. Urgent HRU decreased from median of 4 to 1 (75\% decline). Activity-limited days reduced from a median of 7 to 3.5 (50\% decline). Regular clinic visits increased from 2.5 to 3.5 .

\section{Economic evaluations of $\mathrm{CHW}$ interventions aimed at reducing malnutrition}

Setting Two studies were conducted in Bangladesh [24] and Jamaica [27]. One study was conducted from a provider perspective [27] and the other from a societal perspective [24].

Description of interventions The $\mathrm{CHW}$ interventions focussed on providing nutritional advice [24, 27], monitoring growth [24, 27], screening children for malnutrition [24, 27], and treating malnutrition in the community [24] as well as referring malnourished children to the clinics [27]. The studies were not focussed solely on malnutrition, and CHWs had other tasks including counselling communities on nutrition, health, and sanitation. Furthermore, they used algorithms to deliver community case management of basic childhood illness including diarrhoea and acute respiratory infection [24]. Other tasks included following children who had dropped out of immunization programmes [24]. In the economic evaluation conducted by Melville and colleagues [27], CHWs referred children with diarrhoea to clinics. In the study by Puett and colleagues [24], CHWs provided follow-up visits at home to cases of severe acute malnutrition (SAM) without medical complications. Their activities included distributing a weekly ration of Plumpy'Nut (Nutriset, Malaunay, France) and monitoring growth in children.

In both studies, the training of CHWs was similar in terms of intensity and varied in terms of content. CHWs received an initial training of about 1 week. Thereafter, they received monthly refresher training and ongoing supervisory support to implement the community case management of severe acute malnutrition (SAM).

Results One study measured cost-effectiveness using DALYs [24]. The other study was a partial economic evaluation-costing analysis [27]. Both studies were found to be efficient: one cost-effective [24] and the other [27] of moderate cost in its context.

The community case management of SAM in Bangladesh cost US\$26 per DALY averted, compared with US\$1344 per DALY averted for inpatient treatment. The average cost to participant households for their child to recover from SAM in community treatment was one-sixth that of inpatient treatment [24]. In the study by Melville et al. [27], malnutrition levels as measured by weight for age decreased by $34.5 \%$. Eighty-one percent of the children gained adequate weight over a 2 -year period. The overall cost of the programme per child over the 2-year period was US\$31.1 (annual cost of US\$14.50). 


\section{Economic evaluations of $\mathrm{CHW}$ interventions aimed at improving children's physical health and psychomotor development}

Settings Two economic evaluations identified were conducted in Chile in an extremely poor neighbourhood [26] and in Pakistan [21]. Only one study explicitly stated that it was conducted from the provider perspective [21].

Description of the intervention In the economic evaluation by Aracena et al. [26], the intervention was targeted at adolescent single mothers who conceived a first child between 14 and 19 years old. CHWs (referred to as health educators in the study) under supervision of nurse midwives carried out home visits, and there were at least 12 visits, each $1 \mathrm{~h}$ in duration, from the third trimester until the child was 1 year old. Standard health care for adolescent single mothers consists of 10 prenatal consultations with nurse midwife [26]. The intervention sought to (1) encourage the young women's development of their identity as a woman, adolescent, and mother; (2) help her develop life plans; (3) reinforce her parenting skills; (4) promote basic health care practices for both mother and child; and (5) strengthen the adolescent's relationships with those around her.

In the economic evaluation conducted by Gowani et al. [21], the intervention was delivered using an existing $\mathrm{CHW}$ programme. They delivered an integrated responsive stimulation and nutrition intervention to infants to improve early childhood development. CHWs conducted a combination of monthly group and individual home visits. Group visits were approximately $80 \mathrm{~min}$ each, and home visits ranged from 7 to $30 \mathrm{~min}$.

Results The efficiency of the two intervention studies above was assessed by a cost-effectiveness analysis [21, 26].

Findings from the Aracena et al. study [26] showed only the following outcomes had a statistically significant difference: (1) development of children's language skills, (2) nutritional state of the mother, and (3) mental health of the mother. Cost of standard care was US\$ 50 per adolescent over a period of 15 months, and median cost of intervention for the home visit programme was US\$ 90 per adolescent over a period of 15 months. An investment of US\$ 13.50 for a total of 15 months in the home visit programme results in improved mental health of an adolescent mother.

A cost-effectiveness analysis of the results verifies that early childhood interventions that include responsive stimulation are more cost-effective than a nutrition intervention alone in promoting children's early development. Costs of a responsive stimulation intervention integrated in an existing community-based service providing basic health and nutrition care is approximately US\$4 per month per child [21].

\section{Economic evaluations of $\mathrm{CHW}$ interventions aimed at reducing mortality of neonates, children under five, improving maternal health}

Setting Three identified economic evaluations were conducted in Nepal [23] and two in Pakistan [20, 22]. All three studies were conducted from a provider's perspective.

Description of intervention In the economic evaluation conducted by Borghi et al. [23], the intervention was health service strengthening and this was achieved through training government health staff on essential newborn care and provision of basic supplies and equipment. CHWs convened monthly meetings with women's groups.

In the economic evaluation by Barzgar et al. [20], CHWs delivered primary care and mobilized communities for health. In the economic evaluation by Hafeez et al. [22], CHWs provided education on antenatal care, immunization services, provision of family planning, and basic curative care. Each $\mathrm{CHW}$ house was declared a Health House where people can come in for emergencies and to receive basic advice. $\mathrm{CHW}$ sere also responsible for record keeping and tracking of health indicators. The economic evaluation conducted in Ecuador [25] was done from the provider-patient perspective. CHWs in each community and spent a total of 10 days on vaccination campaign.

Results The cost-effectiveness of the interventions was measured using generic economic evaluation measures, mortality, and disease- or condition-specific measures and intervention process measures.

The average annual cost per woman of reproductive age was US\$ 4.38 (US\$ 5.22 with health service strengthening). The average annual cost per newborn infant was US\$ 22.51 (US\$ 26.82 with health systems strengthening (HSS)). Neonatal deaths averted were US\$ 30.94. Cost per neonatal death averted was US\$ 5801 (US\$ 6912 with HSS). Life years saved per death averted was 27.54. The cost per life year saved was US\$211 (US\$ 251 with HSS). Total life years saved were 852 [23].

The intervention was aimed at reducing infant, child, and maternal mortality within a year and it generated positive perceptions of family planning. This intervention was found to be cost-effective [20]. A 50\% reduction in infant mortality from $130 / 1000$ at baseline to $64 / 1000$ after intervention was observed. An above $50 \%$ reduction in maternal mortality, i.e. 596 per 100000 at baseline to 246 per 100000 after intervention, was also observed. Another outcome measure that had a more than $50 \%$ reduction was infant diarrhoeal deaths. Finally, a more than $97 \%$ reduction in neonatal tetanus was observed. There was no observable impact on low birth weight, malnutrition, and pneumonia. Immunization 
coverage increased by 80,70 , and $100 \%$ in Chakwal, Mastung, and Malir, respectively. Costs per person were US\$ 0.39 for capital costs and US\$ 1.13 per person for recurrent costs.

The national CHW programme was found to be more efficient compared to the status quo. Eighty percent of children were fully immunized under the $\mathrm{CHW}$ programme compared to $47 \%$ in the areas that are not covered by CHWs. Infant mortality rate was $51 \%$ in the $\mathrm{CHW}$-covered areas compared to $39 \%$ in non-CHWcovered areas. Maternal mortality rate was 180 compared to 276 in non-CHW-covered areas. Finally, the average cost of each CHW was US\$570 per year [22].

\section{Discussion}

This review highlights the cost-effectiveness of CHW interventions for health issues that contribute substantially to the ongoing burden of disease in low- and middle-income countries. In addition, this review covers an important topic for human resources for health in particular for health systems in low- and middle-income countries that are trying to scale up interventions to meet various population needs including the emerging non-communicable diseases. There is evidence of costeffectiveness of CHWs interventions in reducing malaria, asthma, and mortality of neonates and children under 5 years of age. Other economic evaluation studies show evidence of cost-effectiveness in improving exclusive breastfeeding, malnutrition, physical health and psychomotor development in children, and maternal health.

This review is based on all four types of economic evaluation-cost-effectiveness, cost utility, cost benefit, and cost minimization analysis. Studies were not excluded on the basis of scale of implementation (i.e. CHW programmes implemented as vertical programmes or integrated). A systematic approach was used to identify and select economic evaluation studies and synthesizing data across studies and assess the quality of the evidence obtained.

\section{Limitations}

It was not possible to pool the studies and conduct a meta-analysis due to a limited number of identified studies per outcome. Furthermore, the economic evaluations we identified had varied measures of economic evaluation outcomes, for instance disease- or conditionspecific outcomes, morbidity, mortality, and generic measures (e.g. disability-adjusted life years (DALY)).

This review focused only on studies published in peerreviewed journals, in English. Thus, a language as well as a publication bias may have been introduced.

Our findings were consistent with Perry and Zulliger [10] only on cost-effectiveness of community-based therapeutic care for children with severe malnutrition, malaria control programmes, perinatal and neonatal care programmes, and child survival programmes with multiple interventions. It is worth noting that even though our findings are consistent with Perry and Zulliger' $\mathrm{s}$ [10] with respect to certain interventions, our evidence was drawn from a different pool of studies. They identified 33 studies whilst the present study included 19 studies. There was only one common study between these two reviews. Perry and Zulliger's [10] review differs in how they have selected studies for inclusion. They appear to have selected studies with bundled interventions. It is difficult to know what type of studies their review selected because the methodology does not have sufficient detail. We specifically excluded any studies that had CHWs as one component and for which one could not directly link the impact of the intervention to CHWs.

To aid decision-making on which interventions are cost-effective and should be implemented in a particular context, economic evaluations in this review used different criterion for assessing cost-effectiveness. These included recommendations from a macro-economic commission, the World Bank, and World Health Organization. The WHO Commission on Macroeconomics and WHOCHOICE threshold guidelines stipulate that an intervention is (1) highly cost-effective if the cost-effectiveness ratio is less than three times gross domestic product per capita, (2) cost-effective if the cost-effectiveness ratio is between one and three times gross domestic product per capita, and (3) not cost-effective if the cost-effectiveness ratio is greater than three time gross domestic product per capita [13]. All interventions were found to be cost-effective or highly cost-effective in their respective countries. The use of these thresholds is methodologically sound in the context of implicit budgets. These thresholds serve as a guide and indicate that a country can potentially invest in that intervention. For instance, the expenditure on health varies between low- and middle-income countries. Thus, these thresholds can be seen as the first level of priority setting. It is crucial for countries to embark on a country-specific priority setting that is informed by their epidemic profile (infant mortality and morbidity), current state of human resources, and availability of human resource.

\section{Implications for future research}

It is somewhat encouraging that there has been an increase in the number of economic evaluations conducted on CHW interventions. However, costs of crucial components of $\mathrm{CHW}$ programmes continue to be poorly reported. These include supervision, start-up costs (i.e. intervention development, recruitment, and initial training), and ongoing training costs. Only 7 out of the 19 included studies reported indirect costs, and only 1 study reported start-up costs. It is this level of detail that will inform national programme planning and budgeting. 
Ten out of 19 studies reviewed in this paper covered a population of 1000 or more participants. The remaining 9 covered a population size ranging from 41 to 726 participants. Majority of these studies were stand-alone interventions. Thus, future research must focus on economic evaluations of multi-country, integrated CHW programmes. Several countries ${ }^{7}$ have national CHW programmes that vary in coverage of the population, number of $\mathrm{CHW}$ trained, and year of initiation of the national programme. These studies can provide insightful lessons on economies of scale and scope that can be achieved or not achieved in CHW programmes operated at a national level.

In the meantime, countries which have decided to initiate or expand their national CHW programmes are faced with the decision of which package of intervention would deliver the best value (i.e. better health outcomes) for their investment. Modelling resource implications for packages of interventions with demonstrated effectiveness using tools like the Lives Saved Tool (LiST) (http://www.livessavedtool.org/) and One Health (http:// www.who.int/choice/onehealthtool/en/) can aid countries to make better informed decisions in the absence of empirical evidence from primary studies.

Given the importance of CHWs in achieving universal health coverage, it is to be expected that research on effectiveness and cost-effectiveness of CHWs will continue. Thus, there is a need for an update review of economic evaluation of CHW intervention in 5 years' time. This review must be a systematic review, include both published and non-published material, and it should be broader in scope. Furthermore, the review should address other conceptual issues such as costing community-based interventions versus costing CHW programmes as well as the importance of community participation or engagement.

\section{Conclusions}

The growing body of evidence for cost-effectiveness of community health workers in improving child health outcomes presents an enormous opportunity for governments in low- and middle-income countries to invest in child survival. However, a stronger evidence base on the cost-effectiveness of specific packages of interventions delivered by community health workers is necessary to guide country health policy and programme implementation.

\section{Endnotes}

${ }^{1} \mathrm{CMAs}$ in this type of economic evaluation comparisons of interventions are only based on costs, and outcomes are assumed to be identical. CMA seeks to answer the question, what is the least costly way to obtain an outcome?

${ }^{2} \mathrm{CEA}$ is of most use in situations where a decision marker, operating with a given budget, is considering a limited range of options within a given field. In a CEA, outcomes are measured in their natural units or life years saved.
${ }^{3} \mathrm{CUA}$ is a form of economic evaluation where the consequences of programmes are adjusted by health state preference scores or utility weights. In general terms, this means that one can assess the quality of life years gained, not just the crude number of years. The most common measure of consequences in CUAs is the quality-adjusted life year.

${ }^{4} \mathrm{CBA}$ is a form of economic evaluation that values consequences of programmes in money terms, so as to make them commensurate with the costs. This is the broadest form of analysis.

${ }^{5} \mathrm{CC}$ is a form of economic evaluation where decisionmakers are presented with treatments or programmes with multiple objectives along with an array of the differential achievements, along each dimension, for the various alternatives. These data can then be presented to the decision-makers, so that they can make their own trade-off between effects.

${ }^{6} \mathrm{~A}$ costing analysis is a partial economic evaluation.

${ }^{7}$ Bangladesh, Thailand, Brazil, Haiti, Ethiopia, Mozambique, Pakistan, and South Africa.

\section{Additional file}

Additional file 1: Search strategy. (DOCX $21 \mathrm{~kb}$ )

\begin{abstract}
Acknowledgements
The authors would like to acknowledge Mandy Maredza for the following input: searching for articles using a search strategy developed by LN and screening articles for inclusion. In addition, Tamara Kredo reviewed an earlier draft of the manuscript which strengthened this paper. The content of the paper, however, remains the responsibility of the authors.
\end{abstract}

\section{Funding}

This review was funded by PEECHi, the Programme for Economic Evaluation of Child and Maternal Health Interventions (grant number D1305910-01), through PRICELESS SA.

\section{Availability of data and materials}

There is no data to share; this article is based on a review of articles published in peer-reviewed journals.

\section{Authors' contributions}

All authors ( $L N, A T$, and $\mathrm{KH}$ ) participated in the design of the study. $L N$ analysed the data and drafted the manuscript, which all authors commented on and approved.

\section{Competing interests}

The authors declare that they have no competing interests.

\section{Consent for publication}

Not applicable.

\section{Ethics approval and consent to participate} Not applicable.

\section{Author details}

${ }^{1}$ Centre for Health Systems and Services Revision, Division of Community Health, Faculty of Medicine and Health Sciences, Francie van Zijl Rylaan/ Drive, Tygerberg 7505, South Africa. ${ }^{2}$ PRICELESS SA, MRC/Wits Rural Public Health and Health Transitions Research Unit (Agincourt), School of Public Health, Faculty of Health Sciences, University of the Witwatersrand,

Johannesburg, South Africa. 
Received: 31 October 2015 Accepted: 15 February 2017 Published online: 28 February 2017

\section{References}

1. Gilson L, et al. National community health worker programs: how can they be strengthened? J Public Health Policy. 1989;10(4):518-32.

2. Ofosu-Amaah V. National experience in the use of community health workers: a review of current issues and problems. Geneva: World Health Organization; 1983.

3. Bhattacharyya $\mathrm{K}$, et al. Community health worker incentives and disincentives: how they affect motivation, retention, and sustainability. Virgian: Basics II; 2001.

4. Lehmann U, Sanders D, Community Health Workers. What do we know about them? The state of the evidence on programmes, activities, costs and impact on health outcomes of using community health workers, Evidence and information for policy, department of human resource for health. Geneva: World Health Organisation; 2007.

5. Bhutta ZA et al. Global experience of community health workers for delivery of health related Millennium Development Goals: a systematic review, country case studies, and recommendations for scaling up. Global health workforce alliance: health workers for all and all for health workers. 2010

6. Lewin S, et al. Lay health workers in primary and community health care for maternal and child health and the management of infectious diseases (Review). The Cochrane Library. 2010. p. 211. Issue 3.

7. Perry HB, Zulliger R, Rogers MM. Community health workers in low-, middle, and high-income countries: an overview of their history, recent evolution, and current effectiveness. Annu Rev Public Health. 2014;35:399-421.

8. Lewin $\mathrm{S}$ et al. Lay health workers in primary and community health care for maternal and child health and the management of infectious diseases. Cochrane Database Syst Rev. Update in progress.

9. Corluka A, et al. Are vaccination programmes delivered by lay health workers cost-effective? A systematic review. Hum Resour Health. 2009;7(1):81

10. Perry $H$, Zulliger R. How effective are community health workers? An overview of current evidence with recommendations for strengthening community health worker programs to accelerate progress in achieving the health-related Millennium Development Goals. Baltimore: John Hopkins Bloomberg School of Public Health; 2012.

11. Briggs $A, O^{\prime} B r i e n ~ B$. The death of cost-minimisation analysis. Health Econ. 2001;10(2):179-84

12. Pegurri E, Fox-Rushby J, Damian W. The effects and costs of expanding the coverage of immunisation services in developing countries: a systematic literature review. Vaccine. 2005;23:1624-35.

13. Alonzo González M, Menendez C, Font F, Kahigwa E, Kimario J, Mshinda H, Tanner M, Bosch-Capblanch X, Alonso PL. Cost-effectiveness of iron supplementation and malaria chlorophyll in the prevention of anemia and malaria among Tanzanian infants. Bull World Health Organ. 2000;78(1):97-107.

14. Tozan $Y$, et al. Prereferral rectal artesunate for treatment of severe childhood malaria: a cost-effectiveness analysis. Lancet. 2010;376(9756):1910-5.

15. Conteh $L$, et al. Cost effectiveness of seasonal intermittent preventive treatment using amodiaquine \& artesunate or sulphadoxine-pyrimethamine in Ghanaian children. PLoS One. 2010;5(8):e12223.

16. Nonvignon J, et al. Is home management of fevers a cost-effective way of reducing under-five mortality in Africa? The case of a rural Ghanaian District La prise en charge à domicile des fièvres est-elle un moyen rentable de réduire la mortalité des enfants en Afrique? Cas d'un district rural du Ghana ¿El manejo domiciliario de la fiebre es una forma costo-efectiva de reducir la mortalidad en menores de cinco años en África? El caso de un distrito rural en Ghana. Trop Med Int Health. 2012;17(8):951-7.

17. Desmond C, et al. Scaling-up exclusive breastfeeding support programmes: the example of KwaZulu-Natal. PLoS One. 2008;3(6):e2454.

18. Pagnoni $F$, et al. A community-based programme to provide prompt and adequate treatment of presumptive malaria in children. Trans R Soc Trop Med Hyg. 1997;91(5):512-7.

19. Chola $L$, et al. Cost of individual peer counselling for the promotion of exclusive breastfeeding in Uganda. Cost Eff Resour Alloc. 2011;9(1):11.

20. Barzgar MA, Sheikh MR, Bile MK. Female health workers boost primary care. World Health Forum. 1997;18(2):202-10.

21. Gowani $\mathrm{S}$, et al. Cost effectiveness of responsive stimulation and nutrition interventions on early child development outcomes in Pakistan. Ann N Y Acad Sci. 2014;1308(1):149-61.
22. Hafeez A, et al. Lady health workers programme in Pakistan: challenges, achievements and the way forward. J Pak Med Assoc. 2011;61(3):210-5.

23. Borghi J, et al. Economic assessment of a women's group intervention to improve birth outcomes in rural Nepal. Lancet. 2005;366(9500):1882-4.

24. Puett $C$, et al. Cost-effectiveness of the community-based management of severe acute malnutrition by community health workers in southern Bangladesh. Health Policy Plan. 2013;28(4):386-99.

25. San Sebastian $M$, et al. Improving immunization coverage in rural areas of Ecuador: a cost-effectiveness analysis. Trop Doct. 2001;31(1):21-4.

26. Aracena $\mathrm{M}$, et al. A cost-effectiveness evaluation of a home visit program for adolescent mothers. J Health Psychol. 2009;14(7):878-87.

27. Melville B, et al. Growth monitoring: the role of community health volunteers. Public Health. 1995;109(2);111-6.

28. Frick KD, Pugh LC, Milligan RA. Costs related to promoting breastfeeding among urban low-income women. J Obstet Gynecol Neonatal Nurs. 2012;41(1):144-50

29. Pugh LC, et al. Breastfeeding duration, costs, and benefits of a support program for low-income breastfeeding women. Birth. 2002;29(2):95-100.

30. Margellos-Anast H, Gutierrez MA, Whitman S. Improving asthma management among African-American children via a community health worker model: findings from a Chicago-based pilot intervention. J Asthma. 2012:49(4):380-9.

31. Morrell CJ, et al. Costs and effectiveness of community postnatal support workers: randomised controlled trial. BMJ. 2000;321;593-8.

32. Muenning P. Designing \& conducting cost-effectiveness analyses in medicine \& health care. 1st ed. 2002. p. 356

33. Morrell $\mathrm{C}$, et al. Costs and benefits of community postnatal support workers: a randomised controlled trial. Health Technol Assess. 2000:4(6):86.

\section{Submit your next manuscript to BioMed Central and we will help you at every step:}

- We accept pre-submission inquiries

- Our selector tool helps you to find the most relevant journal

- We provide round the clock customer support

- Convenient online submission

- Thorough peer review

- Inclusion in PubMed and all major indexing services

- Maximum visibility for your research

Submit your manuscript at www.biomedcentral.com/submit
Biomed Central 